\title{
Banti's Syndrome Presenting as Hematemesis - A Case Report
}

\author{
Aishwarya Ghule ${ }^{1}$, Sourya Acharya ${ }^{2}$, Samarth Shukla ${ }^{3}$, Sunil Kumar ${ }^{4}$, Parth Godhiwala ${ }^{5}$ \\ 1,2,4,5 Department of Medicine, Datta Meghe Institute of Medical Sciences (Deemed to Be \\ University), Wardha, Maharashtra, India. ${ }^{3}$ Department of Pathology, Datta Meghe Institute of \\ Medical Sciences (Deemed to Be University), Wardha, Maharashtra, India.
}

\section{INTRODUCTION}

Massive splenomegaly presenting with hypersplenism, pancytopenia and portal hypertension, without any underlying known cause is known as Banti's syndrome. There are various causes of splenomegaly. When all the known causes of portal hypertension are ruled out, it is termed as Banti's syndrome. This syndrome was discovered by Guido Banti in 1882 and is named after him. Banti's syndrome is also known as idiopathic portal hypertension or non-cirrhotic portal fibrosis. ${ }^{1}$ Banti's syndrome is commonly found in India and Japan than in the West. ${ }^{2}$

There is absence of any haematologic cause, primary hepatic cause or any tumour or mass lesion involving the spleen. Banti had stated that the primary organ involved was spleen and not the liver leading to secondary splenomegaly. Other features include normal liver function tests, varices seen in endoscopy, cytopenia of one or more cell lines, absence of cirrhosis, patent hepatic veins and elevated portal pressure with multiple collaterals. The complications include rupture of varices and massive bleeding. ${ }^{3}$ We report a case of a 20 -year-old male who presented to us with a history of fever for 7 days and one-episode of hematemesis on the day of admission. All known causes of hypersplenism were ruled out and he was diagnosed to have idiopathic massive splenomegaly with portal hypertension and hypersplenism.

\section{PRESENTATION OF CASE}

A 20-years-old male, non-alcoholic came with complaint of one episode of hematemesis about $150 \mathrm{ml} 1$ hour back. There was no history of jaundice, diarrhoea, easy bruising, melaena, distension of abdomen, swelling over feet, cough, altered sensorium, chest pain, palpitations, breathlessness, orthopnea or paroxysmal nocturnal dyspnoea (PND). Patient was not a known case of any chronic illness. He did not have similar complaints in the past.

On general examination patient was well built and well oriented. He was afebrile. His pulse was 92 / min, regular, normal volume and character. Blood pressure was 110 / 70 mmHg. There was no sign of pallor, icterus, clubbing, cyanosis, lymphadenopathy and oedema. Jugular venous pressure (JVP) was normal. On abdominal examination massive splenomegaly was present $13 \mathrm{cms}$ below left costal margin, palpable edge beyond umbilicus; (grade 4) (Figure 1). It was non tender. Liver was not palpable. Abdomen was of normal shape and skin over the abdomen was normal with no dilated veins, scars and no visible pulsations. All signs of liver cell failure were absent.

\author{
Corresponding Author: \\ Dr. Sourya Acharya, \\ Professor, \\ Department of Medicine, \\ DMIMS (Deemed to Be University), JNMC, \\ Wardha, Maharashtra, India. \\ E-mail: souryaacharya72@gmail.com
}

DOI: 10.14260/jemds/2021/161

How to Cite This Article:

Ghule A, Acharya S, Shukla S, et al. Banti's syndrome presenting as hematemesis - A case report. J Evolution Med Dent Sci 2021;10(10):749-751, DOI: 10.14260/jemds/2021/161

Submission 23-10-2020,

Peer Review 14-01-2021,

Acceptance 20-01-2021,

Published 08-03-2021.

Copyright (C) 2021 Aishwarya Ghule et al. This is an open access article distributed under Creative Commons Attribution License [Attribution 4.0 International (CC $B Y 4.0)]$ 

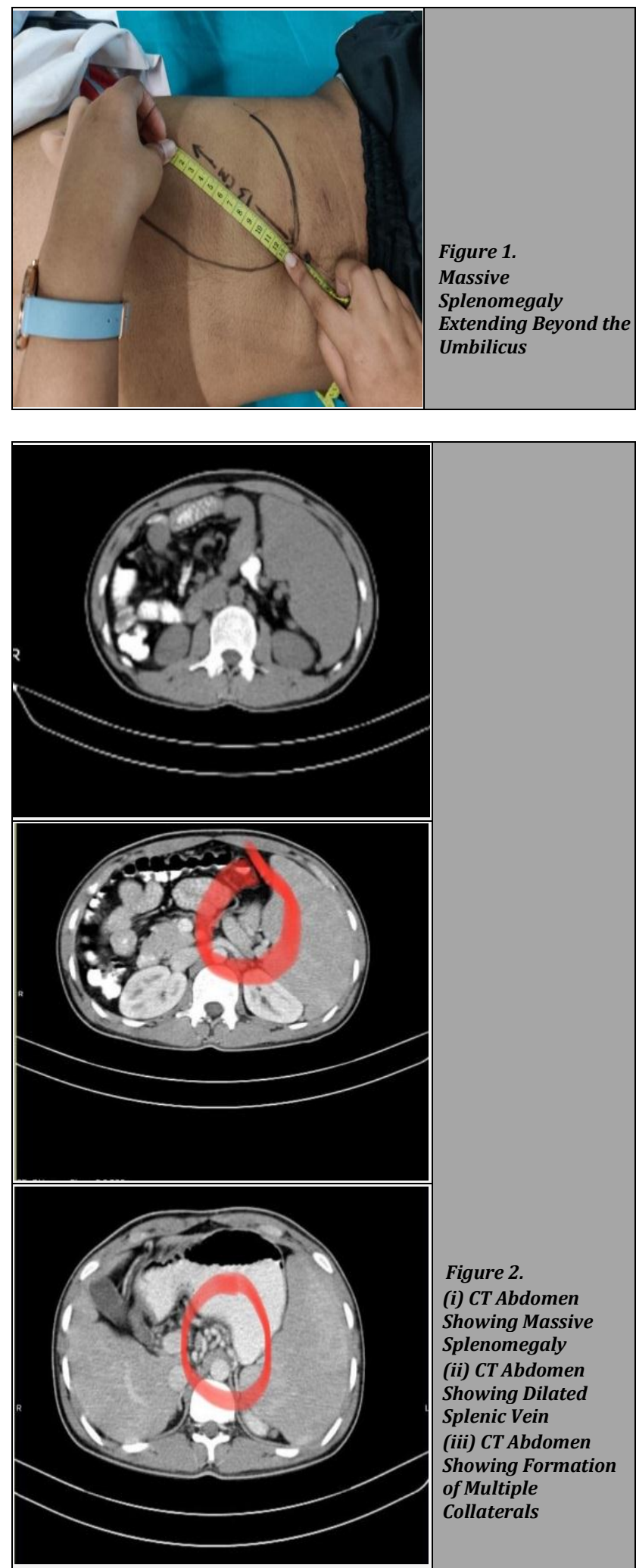

Examination of all other systems was unremarkable. Complete blood count revealed; haemoglobin ( $\mathrm{Hb})-11 \mathrm{gm} \%$, mean corpuscular volume (MCV) was 82, platelet count was 20,000, white blood cell count (WBC) count was 2300. In differential leukocyte count (DLC) lymphocytes were $40 \%$, neutrophils were $50 \%$, eosinophils were $5 \%$, monocytes were $5 \%$ and basophils were $0 \%$. Peripheral smear showed normocytic normochromic red blood cells (RBCs), platelets reduced on smear, antigen presenting cell (APC) - 80,000 cells / cumm, no hemoparasites seen. IgM for malaria was negative.
Kidney function tests and liver function tests were within normal limits. Prothrombin time activated partial thromboplastin time and international normalised ratio (INR) was normal. Urine showed no (RBCs). Lactate dehydrogenase (LDH), erythrocyte sedimentation rate (ESR) and serum ferritin all were within normal limits. Markers for human immunodeficiency virus (HIV), hepatitis B and hepatitis C were negative. Serum copper and serum ceruloplasmin was within normal range.

Ultrasonography of abdomen and pelvis was suggestive of massive splenomegaly measuring $22.8 \mathrm{~cm}$ and an isolated dilated splenic vein measuring $9.8 \mathrm{~mm}$. Computed tomography (CT) scan of the abdomen was suggestive of gross splenomegaly measuring $22 \mathrm{~cm}$, and portal hypertension with multiple varices in the left gastric, lower oesophageal and splenic hilar region. (Figure 2. i, ii, iii.)

Upper gastroendoscopy was suggestive of large oesophageal varices, mild portal gastropathy (PGP) and mild gastric antral vascular ectasia (GAVE). Endoscopic band ligation was done for the varices. (Figure 3)

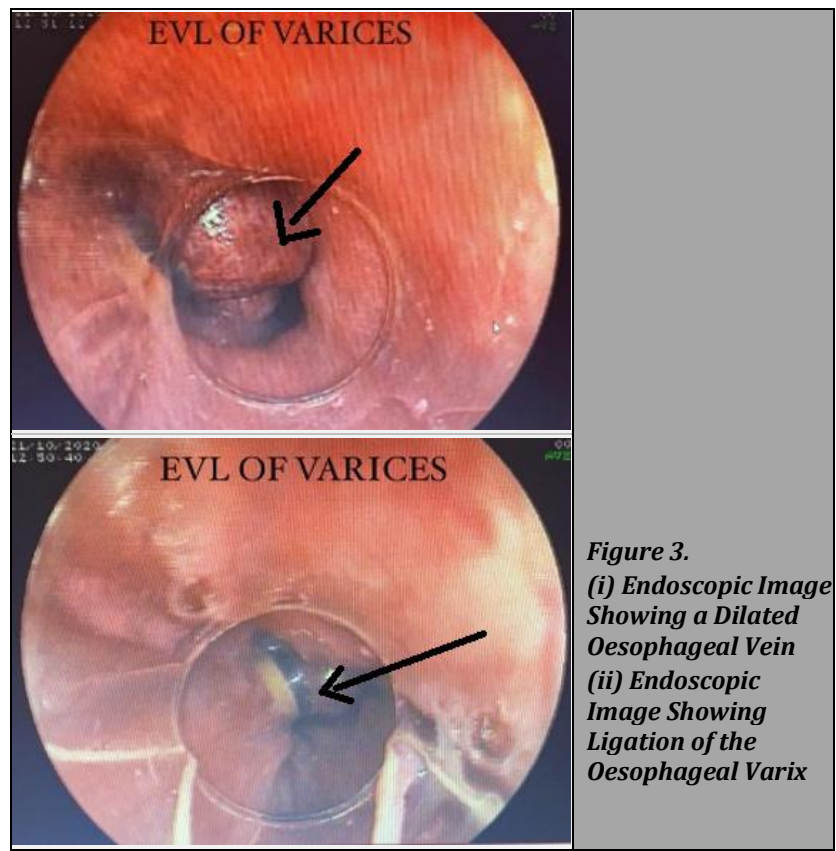

\section{DISCUSSION OF MANAGEMENT}

Patient was started on beta blocker prophylaxis, tablet propranolol $40 \mathrm{mg}$ OD, for portal hypertension. Meanwhile he was also given supportive treatment and plenty of hydration for his dengue infection. Patient improved symptomatically and was discharged.

\section{DISCUSSION}

Banti's syndrome patients present with massive splenomegaly with or without varices. Jaundice rarely develops in these patients. Pancytopenia is due to hypersplenism. ${ }^{4}$ Leukopenia and thrombocytopenia is due to peripheral pooling of blood. Bone marrow is hypercellular. 4,5 Complications like hepatic encephalopathy and ascites rarely develop in these patients. 
Symptomatic hypersplenism is rare in such patients. ${ }^{4}$ Several cases in India, male predominance was found, while in the West and Japan female preponderance is found. Also it affects a more younger group of patients ranging from 25 to 35 years. ${ }^{4}$ Various hypothesis are stated such as hepatitis B infections, various intra-abdominal and systemic infections, abnormalities of clotting of blood and also chronic exposure to substances like arsenic causing fibrosis of small portal veins. 6,7 Several genetic and immune mediated mechanisms are also implicated related to rise in $\mathrm{T}$ helper1 cells and reduction in cytotoxic / suppressor lymphocytes. Association of HLA-DR has been associated with the immunologic process. ${ }^{8}$ Sarin et al. proposed that in genetically predisposed individuals, thrombosis of small and medium branches of the portal vein leads to splenomegaly. The portal and splenic veins are seen as dilated on ultrasonography, and similar finding was seen in

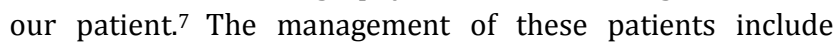
managing hypersplenism and variceal bleeding. In about $95 \%$ of patient's variceal ligation is enough to cease bleeding. Others need shunt surgery. Variceal recurrence has been seen in $20 \%$ of patients, while recurrent bleeding is seen in $3 \%$ of patients. ${ }^{7}$ Surgery is indicated in patients with recurrent variceal bleeding or severe anaemia requiring repeated blood transfusions or recurrent splenic infarcts. Prognosis of Banti's syndrome is very good and after successful ligation of varices the 5 -year survival is said to be $100 \% .^{7}$

\section{CONCLUSIONS}

Banti's syndrome is a diagnosis of exclusion, after all the other causes of portal hypertension and splenomegaly have been ruled out. It has good outcomes after prompt and appropriate treatment. So, Banti's syndrome should be kept in mind in case of hypersplenism, especially in young males and should be treated promptly.

Financial or other competing interests: None.

Disclosure forms provided by the authors are available with the full text of this article at jemds.com.

\section{REFERENCES}

[1] Futagawa S, Fukazawa M, Musha H, et al. Hepatic venography in noncirrhotic idiopathic portal hypertension. Comparison with cirrhosis of the liver. Radiology 1981;141(2):303-9.

[2] Rozenbaum A, Atienza P, Couturier D, et al. Primary hepatoportal sclerosis. Current form of Banti syndrome? Ann Med Interne (Paris) 1988;139(1):52-3.

[3] Okudaira M, Ohbu M, Okuda K. Idiopathic portal hypertension and its pathology. Semin Liver Dis 2002;22(1):59-72.

[4] Sarin SK, Kapoor D. Non-cirrhotic portal fibrosis current concepts and management. J Gastroenterol Hepatol 2002;17(5):526-34.

[5] Dhiman R, Chawla Y, Vasishta R, et al. Non-cirrhotic portal fibrosis (idiopathic portal hypertension) experience with 151 patients and a review of the literature. J Gastroenterol Hepatol 2002;17(1):6-16.

[6] Orozco H, Takahashi T, Garcia-Tsao G, et al. Idiopathic portal hypertension. Rev Invest Clin 1991;43(1):80-6.

[7] Tokushige K, Yamauchi K, Komatsu T, et al. Predominant $\mathrm{T}$ helper I cells in patients with idiopathic portal hypertension. J Gastroenterol Hepatol 2000;15(11):1312-7.

[8] Terada T, Nakanuma Y, Obata H. HLA-DR expression on the microvasculature of portal tracts in idiopathic portal hypertension. Immunohistochemical characteristics and relation to portal phlebosclerosis. Arch Pathol Lab Med 1991;115(10):993-7. 